\title{
Vestibular System Eletrophysiology: An Analysis of the Relationship between Hearing and Movement
}

\author{
Aline Cabral de Oliveira1,2@ Luana Soares Honorato de Souza ${ }^{2}$ Carlos Raphael Araújo Daniel2,3(0) \\ Priscila Feliciano de Oliveira ${ }^{2,40}$ Liliane Desgualdo Pereira ${ }^{5}$ \\ ${ }^{1}$ Department of Speech, Language and Hearing Sciences, \\ Universidade Federal de São Paulo, São Paulo, SP, Brazil \\ ${ }^{2}$ Department of Speech, Language and Hearing Sciences, \\ Universidade Federal do Sergipe, Lagarto, SE, Brazil \\ ${ }^{3}$ Department of Statistics and Actuarial Sciences, Universidade \\ Federal do Sergipe, Lagarto, SE, Brazil \\ ${ }^{4}$ Department of Speech, Language and Hearing, Universidade Federal \\ do Sergipe, São Cristóvão, SE, Brazil \\ ${ }^{5}$ Department of Speech, Language and Hearing Sciences, Escola \\ Paulista de Medicina, Universidade Federal de Sâo Paulo, São Paulo, \\ SP, Brazil \\ Int Arch Otorhinolaryngol 2022;26(2):e272-e277. \\ Address for correspondence Priscila Feliciano de Oliveira, PhD, \\ Department of Speech, Language and Hearing Sciences, Universidade \\ Federal do Sergipe. Av. Marechal Rondon, s/n, São Cristóvão, SE, \\ 49100-000, Brazil (e-mail: oliveirapriscila@hotmail.com).
}

\begin{abstract}
Keywords

- vestibular evoked myogenic potentials

- hearing

- postural balance

Introduction Knowledge about the positive effects that music and dance bring, in its various forms, to the healthy human brain, is important not only in the context of basic neuroscience but may also strongly affect practices in neurorehabilitation.

Objective To verify the relationship between hearing and movement and, specifically, to analyze the interference of professional dance practice and formal musical training in the magnitude of the vestibule-cervical and vestibular reflexes.

Method The sample consisted of 92 subjects, aged between 18 and 35 years old, 31 professional musicians, 31 ballet dancers, and 30 control subjects. Only subjects with normal hearing sensitivity were included. Cervical vestibular evoked myogenic potential (cVEMP) was recorded in the sternocleidomastoid muscle, and ocular vestibular evoked myogenic potential (oVEMP) was recorded in the lower oblique muscle of the eye, using tone-bursts $(500 \mathrm{~Hz})$. Analysis of variance (ANOVA) or Kruskall-Wallis tests were performed.

Results The CVEMP presented earlier and higher amplitude waves when recorded in the group of dancers, with a significant difference between all tested groups for latency and amplitude of the $\mathrm{N} 23$ wave; the comparison was restricted between dancers and control groups, with no difference between ballet dancers and musicians. The N1 wave of the oVEMP presented lower latencies in dancers than in musicians and controls $(p=0.001)$. No significant differences were found between the groups for the P1 wave. Conclusion Greater magnitudes of vestibule-cervical reflex responses and faster vestibule-ocular reflex responses were observed in dancers. Dance practice provides greater development of the vestibular system, but musical training also contributes to the magnitude of these responses.
\end{abstract}

received

May 19,2020

accepted

December 7, 2020

published online

September 21, 2021
DOI https://doi.org/ $10.1055 / \mathrm{s}-0041-1726044$. ISSN 1809-9777.

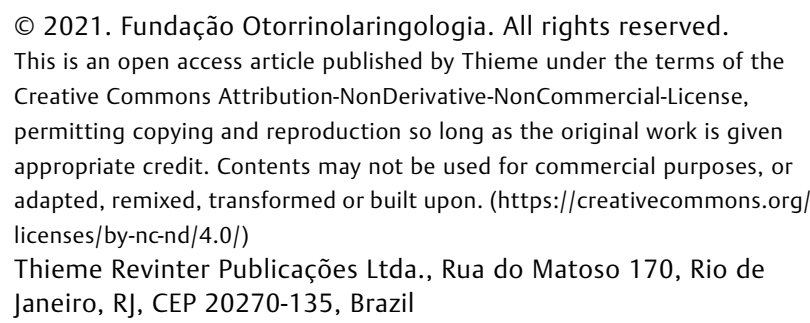

(c) 2021. Fundação Otorrinolaringologia. All rights reserved. This is an open access article published by Thieme under the terms of the Creative Commons Attribution-NonDerivative-NonCommercial-License, permitting copying and reproduction so long as the original work is given appropriate credit. Contents may not be used for commercial purposes, or adapted, remixed, transformed or built upon. (https://creativecommons.org/ licenses/by-nc-nd/4.0/) Thieme Revinter Publicações Ltda., Rua do Matoso 170, Rio de Janeiro, RJ, CEP 20270-135, Brazil 


\section{Introduction}

The integration between hearing and movement occurs at various levels of the nervous system, including the dorsal cochlear nucleus (DCN) and the posterior part of the parietal cortex (PPC). ${ }^{1-3}$ All models of rhythmic movement are centered on the interactions between the posterior part of the parietal cortex, the cerebellum, and the prefrontal cortex. ${ }^{4}$ The cerebellum, a large vestibular entry vessel, has been linked to auditory rhythm. ${ }^{5}$ Some researchers have observed the relationship between music and movement, and others have reported that music may have evolved from physical movement. ${ }^{4,6}$

Several imaging studies demonstrate that the brain regions responsible for synchronized movement are modulated by the auditory metric structure. ${ }^{7-9}$ Body movement involves different types of sensorimotor information, including motor planning, tactile and proprioceptive stimuli, and vestibular inputs. $^{10}$

To learn dance technique, initial reception, memory, and processing of the execution of the movement integrated with the musical rhythm are necessary. ${ }^{11}$ This way, music makes us move, and feeling the rhythm is an interactive process: listening to a rhythm evokes physical movement, and the resulting vestibular stimulation also influences the auditory interpretation of that rhythm. In other words, not only does the musical rhythm activate motor areas of the brain, but movement can improve hearing. ${ }^{12}$

The act of playing a musical instrument - the violin, for example - is a highly complex task. The whole body and various sensory systems are involved and need to be coordinated with a high degree of synchrony and precision. The arms support the violin and move the bow, the fingers of the left hand press the strings, and the somatosensory perception feedback on the posture of the body and the fingertips happens, in a constant and integrated way. The auditory system analyzes the accuracy of the sounds, and the auditory feedback is used to improve its quality; while the visual system checks the musical score and translates these musical symbols into meaningful information involved in the production of music. In addition to the motor and sensory systems, memory, attention, and emotion are involved. However, not only is the musician's brain active, but also the listener's. Even the passive act of listening to music activates a wide range of cortical functions: perception and analysis of pitch, timbre, harmonics, rhythms, metronomes, and structures of a higher order, such as attention and memory systems and emotional responses to the music heard. ${ }^{9,13}$

During musical performance, sensory stimuli (auditory, visual and proprioceptive) and motor commands (articulatory, respiratory, and limb coordination) are integrated. ${ }^{14}$ Musicians usually use body gestures to express the time or the sensation of movement in music. ${ }^{12}$ In musical rhythm training, most of the time, the perception of the beat in the body is initially worked through body movements, and then the rhythm is internalized in an auditory code, which may involve a motor representation, that is, the rhythm is incorporated and expressed through movement. ${ }^{15}$
This incorporated rhythm method used in the teaching of advanced music is based on the interaction between the movement of the body and the auditory perception of the musical beat; "listening inspires movement while moving guides and informs listening". In other words, "how we move will influence what we hear"15.

Studies show that the musician's brain has functional adaptations for musical processing and timbre, ${ }^{16}$ in addition to specializations for the visual, motor, and cerebellar regions. ${ }^{17}$

Therefore, knowledge about the positive effects that music and dance bring, in its various forms, to the healthy human brain, is important not only in the context of basic neuroscience but may also strongly affect practices in neurorehabilitation. ${ }^{13,18}$

Dance practice provides greater cortical reorganization and is supposed to be more complex than that developed using isolated therapeutic exercises (auditory training and vestibular rehabilitation), due to their multimodal nature. Also, music and dance can be a very rewarding and positive experience, which can increase the motivation and cooperation in training of the patients and, consequently, optimize therapeutic effects. Therefore, it is possible to modify/readjust the proposal for auditory and vestibular rehabilitation disseminated in current clinical practice.

Thus, the objective of the present study was to verify the relationship between hearing and movement and, specifically, to analyze the interference of professional dance practice and formal musical training in the magnitude of the vestibulocervical and vestibulo-ocular reflexes.

\section{Methods}

The present work is a cross-sectional analytical observational study developed at Federal University of Sergipe. The protocol for this research is based on Resolution 466/2012 of the National Health Council of the Ministry of Health for studies with human beings, and was approved by the Research Ethics Committee (REC), under the number 2.427.601 issue.

Aged between 18 and 35 years old, 92 young adult subjects were evaluated. They were matched by gender and years of schooling, and were distributed in the following groups $(G)$, namely: G1-31 subjects with professional musical training; G2-31 professional ballet dancers; and G3-30 subjects without professional musical training and not dancers, called Control.

The following inclusion criteria were applied for the entire sample: age group between 18 and 35 years old, of both genders; right-handed; normal hearing sensitivity, that is, hearing thresholds $\leq 20 \mathrm{dBHL}$ (ANSI -1969), with differences between the ears by a frequency $\leq 10 \mathrm{~dB}$; normal speech recognition index (SRI), and normal or type A tympanogram with the presence of acoustic reflexes. The exclusion criteria were: a history of exposure to occupational or leisure noise; the presence of a wax stopper (cerumen) or of a foreign body that interferes with the visualization of the external auditory canal; ear surgery; more than three ear infections in the current year; use of ototoxic medication; presence of tinnitus, dizziness, vertigo or other cochlear-vestibular changes; presence of systemic changes that may contribute to cochleovestibular 
diseases, such as diabetes, high blood pressure, etc.; cervical, visual (uncorrected), and/or proprioceptive changes.

Specifically, each group was formed based on: G1-presenting at least 3 years of professional music education and not practicing dance frequently and/or being a percussionist; G2-at least 3 years of professional ballet practice; G3-not being a professional in music and dance characterized by self-declaration according to frequent or occasional exposure to music.

Initially, a questionnaire (anamnesis) was applied, including questions about personal data, hearing history, and hearing health to screen the participants. Soon after, the research protocol was explained verbally, and then the free and informed consent term (FICT) was obtained. The volunteers who agreed to participate in the present research were submitted to basic audiological evaluation (pure tone audiometry, logoaudiometry, and immittance testing), which was intended to screen subjects with the presence of hearing disorders, excluding them from the study. After screening the subjects, tests for cervical vestibular evoked myogenic potential (cVEMP) and for ocular vestibular evoked myogenic potential (oVEMP) were performed.

Interacoustics EP25 equipment (Interacoustics, Middedlfart, Denmark) was used to perform the VEMP exams (cVEMP and oVEMP). In both cases, the sound stimuli were presented by ER-3A insertion headphones (3M Auditory Systems; Phoenix, AZ, Arizona, United States), and the records were performed using superficial electrodes placed on the skin and fixed with adhesive tape after cleaning with abrasive paste, alcohol, and cotton, with previous use of electrolytic paste. To obtain the cVEMP record, the active electrode was placed on the upper half of the sternocleidomastoid muscle, ipsilateral to the stimulation; the reference electrode was placed on the anterior border of the ipsilateral clavicle, and the ground electrode was placed on the frontal midline. After placing the electrodes, the impedance was evaluated between the noninverting $\mathrm{x}$ ground electrodes and between the inverter $\mathrm{x}$ ground electrodes, allowing, therefore, an impedance between the electrodes of up to $3 \mathrm{k} \Omega$ and of each isolated electrode of 5 $\mathrm{k} \Omega$. During the test, the patient remained seated, with maximum lateral rotation of the head to the contralateral side to the stimulus, and maintained tonic contraction of the muscle at 60 to $80 \mu \mathrm{V}$. The stimulus was initiated by the right gauge and, later, repeated in the left gauge. The responses were replicated, that is, recorded twice on the right side and twice on the left side. One-hundred tone-bursts stimuli (rate of $5 \mathrm{~Hz}$ ), frequency of $500 \mathrm{~Hz}$, at an intensity of $95 \mathrm{dBHL}$, using a bandpass filter from 20 to $2.000 \mathrm{~Hz}$, with amplification of 10 to 25 microvolts per division. The records were made in $50 \mathrm{~ms}$ windows. For the analysis of the cVEMP responses, initially, the first negative and positive peaks were marked, $\sim 13$ and $23 \mathrm{~ms}$, respectively, and then, the latency and amplitude parameters of the p13 and n23 waves were verified.

The procedures used in the oVEMP test were similar to those of cVEMP, and presented the following exceptions:

- Regarding the configuration of the electrodes, the active electrode should be positioned under the lower oblique muscle of the eye; the reference electrode below the active electrode at a distance of 1 to $2 \mathrm{~cm}$, and the ground electrode on the forehead;

- During the recording, the participants were instructed to keep their eyes in the superomedial direction ${ }^{19}$;

- The threshold for the red LED (light-emitting diode) should be between 4 and $6 \mathrm{mV}$;

- Mean calculation of 512 stimuli;

- Bandpass filter from 0.05 to $500 \mathrm{~Hz}$.

\section{Statistical Model}

Descriptive statistics techniques were applied, and the results are expressed in the form of tables and illustrative graphics. The normality of the samples was observed using the Kolmogorov-Smirnov or the Shapiro-Wilk tests. Depending on the normality, to detect differences between the groups in amplitude and latency of the cVEMP and oVEMP tests, the analysis of variance (ANOVA) test or the Kruskall-Wallis test was applied. Alpha values were considered significant when $<0.05$. The established beta value was 0.1 . The software used to make the calculations was IBM SPSS Statistics for Windows, version 21.0 (IBM Corp., Armonk, NY, USA).

\section{Results}

The sample was composed of 92 subjects: 31 musicians (G1), 31 dancers (G2), and 30 control subjects (G3). Groups 1 and 2 were composed of 15 men (48.4\%) and 16 women (51.6\%) each; while G3 presented 15 subjects per gender.

The cVEMP (wave N23) showed earlier waves with greater amplitudes when registered in the group of dancers. Using the ANOVA test or the Kruskall-Wallis test, there was a significant difference between groups G1, G2, and G3 for latency and amplitude of the N23 wave. When performing pairwise analysis, the latency values of N23, through the Tukey test, found a difference that was restricted to the comparison between dancers and controls $(p=0.042)$, with no difference between dancers and musicians $(p=0.483)$. The amplitude values of N23 were greater for dancers, and the musicians showed smaller waves than the control group, as shown in -Table $\mathbf{1}$ and -Figure $\mathbf{1}$.

In the analysis of the oVEMP responses between groups $\mathrm{G} 1, \mathrm{G} 2$, and $\mathrm{G} 3$, we found that the N1 wave has lower latencies for dancers than for musicians and controls $(p=0.001)$; no significant differences were found between groups for the P1 wave. - Table 2

\section{Discussion}

In the present study, lower latencies and greater amplitudes of the N23 wave were found in the cVEMP analysis for the group of dancers, with a significant difference in their comparison with the control group. These findings are consistent with the literature, reaffirming the improvement in the plasticity of the vestibulocervical pathway, which, through regular training, as happens with dancers, makes the otolithic organs more receptive and with better 
Table 1 Significance value when comparing cVEMP wave latency and amplitude values between groups (G1; $n=31)$, (G2; $n=31)$, $(\mathrm{G} 3 ; n=30)$

\begin{tabular}{|c|c|c|c|c|c|}
\hline \multirow{2}{*}{$\begin{array}{l}\text { cVEMP } \\
\text { waves }\end{array}$} & \multirow[t]{2}{*}{ Variable } & \multicolumn{3}{|l|}{ Groups } & \multirow[t]{2}{*}{ p-value } \\
\hline & & G1 & G2 & G3 & \\
\hline \multirow[t]{2}{*}{ P13 } & Latency & $12.82 \pm 3.66$ & $12.65 \pm 3.78$ & $13.47 \pm 3.59$ & $0.49^{*}$ \\
\hline & Amplitude & $5.71 \pm 5.47$ & $5.81 \pm 4.56$ & $6.15 \pm 5.88$ & $0.88^{* *}$ \\
\hline \multirow[t]{2}{*}{$\mathrm{N} 23$} & Latency & $19.92 \pm 4.34$ & $18.96 \pm 3.84$ & $21.33 \pm 4.76$ & $0.019 * \mathrm{~A}$ \\
\hline & Amplitude & $9.92 \pm 7.95$ & $12.91 \pm 6.81$ & $10.24 \pm 8.56$ & $0.004^{* * A}$ \\
\hline
\end{tabular}

Statistics model: Analysis of variance (ANOVA) and Kruskall-Wallis tests. Standard deviation values $\left( \pm\right.$ SD) $\left({ }^{*}\right)$ ANOVA $\left({ }^{* *}\right)$ Kruskall-Wallis test, ${ }^{\text {A }}$ significant $p$-value $(\leq 0.05)$.

Abbreviations: cVemp, cervical vestibular evoked myogenic potential; G1, professional musical training group $(n=31)$; $G 2$, professional ballet dancers group ( $n=31)$; G3, control group $(n=30)$; N23, negative peak 23; P13, positive peak 13.
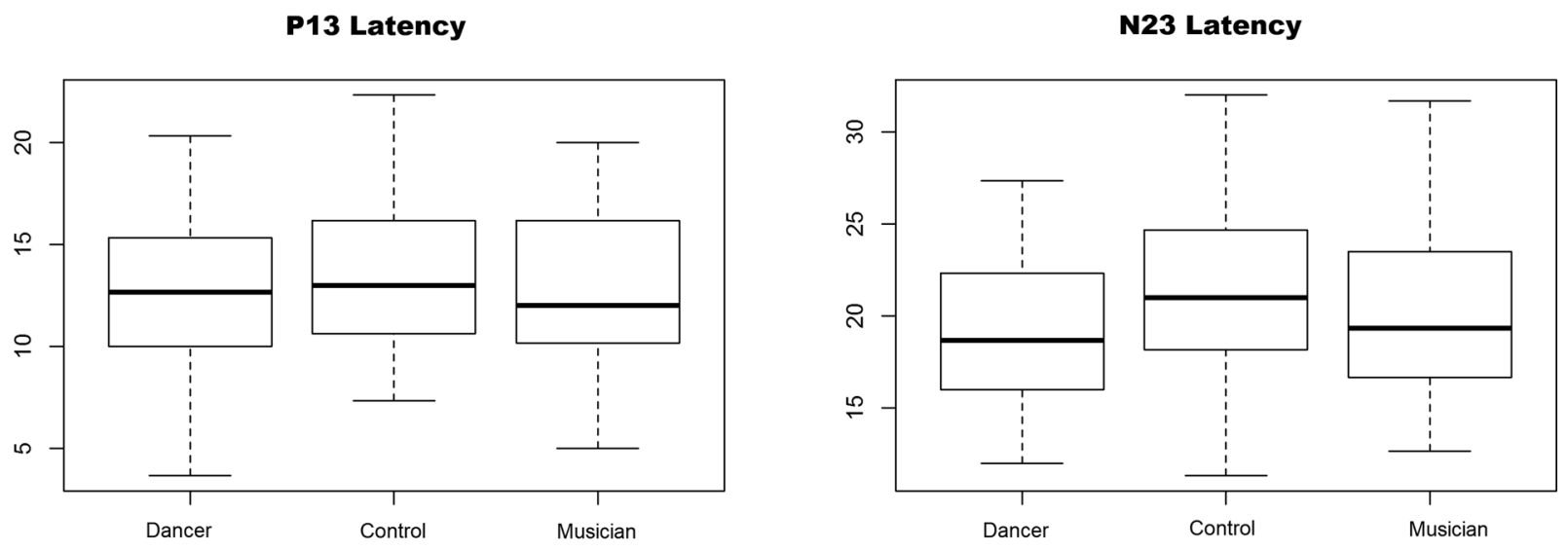

P13 Amplitude

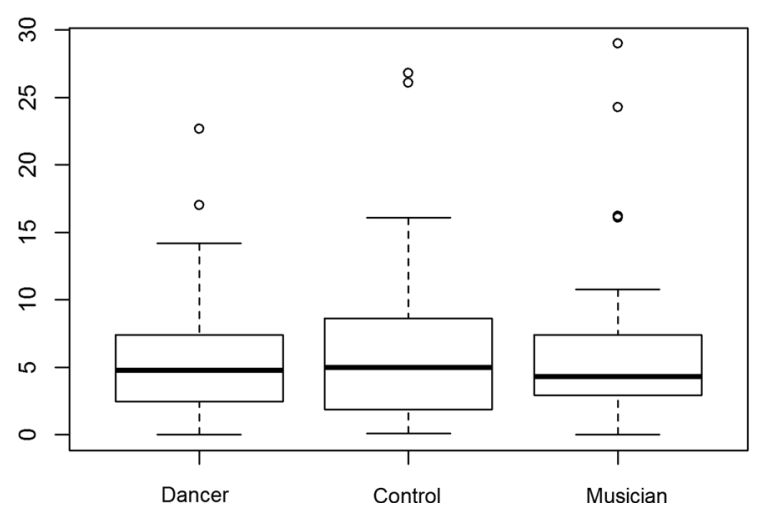

N23 Amplitude

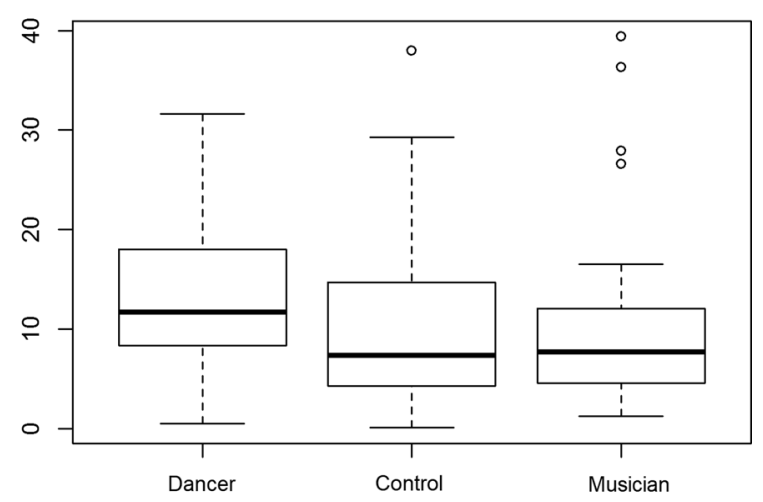

Fig. 1 Boxplot distribution of the latency and amplitude values of the cVEMP waves between the groups. ( $n=31 ; G 1$ musicians), ( $n=31 ; G 2$ dancer), ( $n=30 ; \mathrm{G} 3$ control). Values expressed with standard deviation $( \pm S D)$.

performance in their functions, resulting in an improvement in the responses of these potentials. ${ }^{20}$

The improvement in the plasticity of the vestibulocervical pathway in dancers can be caused by the increased sensitivity of the otolith during constant stimulation, which allows an increased deflection, improving the coupling of the stereocilia and, therefore, providing morphological changes/adaptations in the hair cells of the otolith and faster neural synapses. ${ }^{21}$

Studies on the analysis of the effects of dance on the magnitude of the cervical vestibular reflex also obtained similar results to the present study, demonstrating that regular dance training allows the development of the sacculocollic pathway, improving the speed and quality of responses of the vestibulocervical reflex, contributing to an improvement in the balance of the subjects. ${ }^{22}$

However, in the analysis of the N23 latencies of cVEMP, the average values of the dancers were verified as similar to those of the musicians. These findings can be justified by the integration of sensory stimuli and motor commands that take place during musical performance ${ }^{14}$, in addition to the 
Table 2 P-values referring to the comparison of the latency and amplitude values of the oVEMP waves, between the groups $(G 1 ; n=31),(G 2 ; n=31),(G 3 ; n=30)$

\begin{tabular}{|l|l|l|l|l|l|}
\hline \multirow{2}{*}{ oVEMP waves } & Variables & Groups & \multirow{2}{*}{ p-value } \\
\cline { 3 - 6 } & & G1 & G2 & G3 & \\
\hline N1 & Latency & $11.16 \pm 2.53$ & $10.22 \pm 2.42$ & $11.69 \pm 2.10$ & $0.001^{\mathrm{A}}$ \\
\cline { 2 - 6 } & Amplitude & $3.94 \pm 3.32$ & $4.40 \pm 4.54$ & $3.33 \pm 2.73$ & 0.88 \\
\hline P1 & Latency & $14.84 \pm 2.18$ & $15.51 \pm 3.07$ & $15.84 \pm 2.53$ & 0.54 \\
\cline { 2 - 6 } & Amplitude & $4.00 \pm 3.65$ & $6.02 \pm 6.07$ & $4.57 \pm 3.43$ & 0.33 \\
\hline
\end{tabular}

Statistics model: Analysis of variance (ANOVA) and Kruskall-Wallis tests. Standard deviation values $\left( \pm\right.$ SD) $\left(^{*}\right)$ ANOVA $\left({ }^{* *}\right)$ Kruskall-Wallis test, ${ }^{\text {A }}$ significant $p$-value $(\leq 0.05)$.

Abbreviations: G1, professional musical training group $(n=31)$; G2, professional ballet dancers group $(n=31)$; G3, control group ( $n=30)$; N1, negative peak 1; oVemp, ocular vestibular evoked myogenic potential; P1, positive peak 1.

anatomical relationship between hearing and movement existing at various levels of the nervous system. ${ }^{1-3}$

Despite the amplitude values of N23 being greater in the control group than in the musicians, this parameter has a high interference of the electromyographic tension level and is not directly related to a greater development of the saculecervical reflex. The findings related to latency are directly related to the assessment of the magnitude of this reflex. ${ }^{23}$

Another way to observe the interaction between the auditory and vestibular systems is through ototoxic events, in which damage can compromise the two systems concomitantly and can manifest itself as hearing loss, ear fullness, and hyperacusis, as well as vertigo, dizziness, and tinnitus. This can be justified by their anatomical proximity and functional peculiarity (they are irrigated by the labyrinthine artery, innervated by the vestibulocochlear nerve, and present the same liquids circulating within their cavities - endolymph and perilymph), which allows the interaction between the systems. ${ }^{24,25}$

The fluid imbalance present in the otoliths, through prolonged exposure to noise, was found in individuals with hearing loss induced by high sound pressure levels (PAINPSE), which had prolonged latencies and reduced amplitudes in CVEMP. ${ }^{25,26}$

Studies with adults who had congenital hearing loss show that the saccule and the utricle (assessed by cVEMP and oVEMP) are affected in cases of profound losses, resulting in vestibular dysfunction. Thus, it appears that the damage can extend from one system to the other and justify a higher prevalence of vestibular disorders in deaf children than in healthy children. ${ }^{27,28}$

Regarding the oVEMP findings, significant differences were found between the groups only for the N1 wave, with lower latencies in dancers than in musicians and controls. Several studies have confirmed that the $\mathrm{N} 1$ component is of vestibular origin, resulting specifically from an otolithic-saccular response. $^{20,29,30}$ Some authors report that of the two oVEMP waves, only $\mathrm{N} 10$ is absent in patients with vestibular disorders and present in subjects with hearing loss and normal vestibular function, which suggests that the posterior waves may be involved with nonvestibular components. ${ }^{29}$ Thus, faster responses in the vestibulo-ocular reflex can be justified, as the dance practice significantly contributes to improving

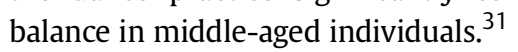

Differently from the findings of the present study, a research carried out with 16 subjects, 8 dancers (Egyptian dance) and 8 nondancers, found no differences in the latency or amplitude parameters of cVEMP or oVEMP between the groups. These findings can be explained, as the studies considered different dance modalities and the authors reported that, possibly, the dance style practiced by the group evaluated in the study did not contribute to the improvement of the sacculocollic and otolithic-ocular plasticity. ${ }^{32}$

\section{Conclusion}

Greater magnitudes of responses were observed in the vestibulo-cervical reflexes, as well as faster responses in the vestibulo-ocular reflex of the dancers. Dance practice provides greater development of the vestibular system, but musical training also contributes to the magnitude of these responses, influencing the plasticity of the vestibular system.

These findings contribute to reinforce the existence of the relationship between hearing and movement, since, through stimulation of the auditory system in musical training, stimulation of the vestibular system was also observed.

The present work contributes, in a relevant way, to improve the knowledge about sensorymotor integration (auditory processing and body movement), and to fill an existing gap regarding the scarcity of data observed in the specialized literature with an approach to the theme: the use of electrophysiology of the vestibular system for analyzing the relationship between hearing and movement.

The limitation of the present study is that we do not know if other types of dance, in addition to classical ballet, cause this degree of contribution to the vestibular system, since classical ballet is of high performance. But our objective was precisely to observe the impact of the best type of motor stimulus to the vestibular system.

Conflict of Interests

The authors have no conflict of interests to declare.

\section{References}

1 Oertel D, Young ED. What's a cerebellar circuit doing in the auditory system? Trends Neurosci 2004;27(02):104-110 
2 Bremmer F. Navigation in space-the role of the macaque ventral intraparietal area. J Physiol 2005;566(Pt 1):29-35

3 Schlack A, Sterbing-D’Angelo SJ, Hartung K, Hoffmann K-P, Bremmer F. Multisensory space representations in the macaque ventral intraparietal area. J Neurosci 2005;25(18):4616-4625

4 Todd NPM, Lee CS, O'Boyle DJ. A sensorimotor theory of temporal tracking and beat induction. Psychol Res 2002;66(01):26-39

5 Molinari M, Leggio MG, De Martin M, Cerasa A, Thaut M. Neurobiology of rhythmic motor entrainment. Ann N Y Acad Sci 2003; 999:313-321

6 Trainor L. Do preferred beat rate and entrainment to the beat have a common origin in music? Empir Musicol Rev 2007;2:17-20

7 Chen JL, Zatorre RJ, Penhune VB. Interactions between auditory and dorsal premotor cortex during synchronization to musical rhythms. Neuroimage 2006;32(04):1771-1781

8 Lahav A, Saltzman E, Schlaug G. Action representation of sound: audiomotor recognition network while listening to newly acquired actions. J Neurosci 2007;27(02):308-314

9 Zatorre RJ, Chen JL, Penhune VB. When the brain plays music: auditory-motor interactions in music perception and production. Nat Rev Neurosci 2007;8(07):547-558

10 Vanderah TW, Douglas JG. Hearing and balance: the eighth cranial nerve. In: Vanderah TW, Douglas JG, eds. Noltès The Human Brain : An Introduction to Its Functional Anatomy. Philadelphia: Elsevier; 2016:348-82

11 Năstase VD. The role of sensations, perceptions and representations in learning dance sport. Procedia Soc Behav Sci 2012; 51:957-960

12 Thompson WF, Graham P, Russo FA. Seeing music performance: Visual influences on perception and experience. Semiotica 2005; 156:177-201

13 Pantev C, Herholz SC. Plasticity of the human auditory cortex related to musical training. Neurosci Biobehav Rev 2011;35(10): 2140-2154

14 Ellis RJ, Norton AC, Overy K, Winner E, Alsop DC, Schlaug G. Differentiating maturational and training influences on fMRI activation during music processing. Neuroimage 2012;60(03): 1902-1912

15 Juntunen ML, Hyvonen L. Embodiment in musical knowing: How body movement facilitates learning with Dalcroze Eurhythmics. Br J Music Educ 2004;21:199-214

16 Wong PCM, Skoe E, Russo NM, Dees T, Kraus N. Musical experience shapes human brainstem encoding of linguistic pitch patterns. Nat Neurosci 2007;10(04):420-422

17 Gaser C, Schlaug G. Brain structures differ between musicians and non-musicians. J Neurosci 2003;23(27):9240-9245
18 Kiefer AW, Riley MA, Shockley K, et al. Multi-segmental postural coordination in professional ballet dancers. Gait Posture 2011;34 (01):76-80

19 Chihara Y, Iwasaki S, Ushio M, Murofushi T. Vestibular-evoked extraocular potentials by air-conducted sound: another clinical test for vestibular function. Clin Neurophysiol 2007;118(12): 2745-2751

20 Swathi V, Kumar KS. Influence of dance training on sacculocollic pathway: vestibular evoked myogenic potentials (VEMP) as an objective tool. J Evol Med Dent Sci 2013;2:7747-7754

21 Bruyneel AV, Mesure S, Paré JC, Bertrand M. Organization of postural equilibrium in several planes in ballet dancers. Neurosci Lett 2010;485(03):228-232

22 Oliveira-Barreto AC, Menezes PL, Feitosa ABL, et al. Dancing effects on the magnitude of the vestibular-cervical reflex. Otolaryngol Head Neck Surg 2017;2:1-3

23 Hall JW. New Handbook for Auditory Evoked Responses. Boston: Pearson Education; 2006

24 Walicka-Cupryś K, Przygoda Ł, Czenczek E, et al. Balance assessment in hearing-impaired children. Res Dev Disabil 2014;35(11): 2728-2734

25 Wang Y-P, Young Y-H. Vestibular-evoked myogenic potentials in chronic noise-induced hearing loss. Otolaryngol Head Neck Surg 2007;137(04):607-611

26 Dalgıç A, Yılmaz O, Hıdır Y, Satar B, Gerek M. Analysis of Vestibular Evoked Myogenic Potentials and Electrocochleography in Noise Induced Hearing Loss. J Int Adv Otol 2015;11(02):127-132

27 Lin B-Y, Young Y-H. Assessing residual vestibular function in adults with congenital hearing loss. Eur Arch Otorhinolaryngol 2016;273(12):4209-4214

28 Jacot E, Van Den Abbeele T, Debre HR, Wiener-Vacher SR. Vestibular impairments pre- and post-cochlear implant in children. Int J Pediatr Otorhinolaryngol 2009;73(02):209-217

29 Iwasaki S, Smulders YE, Burgess AM, et al. Ocular vestibular evoked myogenic potentials to bone conducted vibration of the midline forehead at $\mathrm{Fz}$ in healthy subjects. Clin Neurophysiol 2008;119(09):2135-2147

30 Wang S-J, Jaw F-S, Young Y-H. Ocular vestibular-evoked myogenic potentials elicited from monaural versus binaural acoustic stimulations. Clin Neurophysiol 2009;120(02):420-423

31 Federici A, Bellagamba S, Rocchi MBL. Does dance-based training improve balance in adult and young old subjects? A pilot randomized controlled trial. Aging Clin Exp Res 2005;17(05):385-389

32 Sinha SK, Bohra V, Sanju HK. Comparison of Cervical and Ocular Vestibular Evoked Myogenic Potentials in Dancers and NonDancers. Audiology Res 2013;3(01):e6 